\title{
Gelation of charged bio-nanocompartments induced by associative and non-associative polysaccharides
}

\author{
Filipe E. Antunes ${ }^{\mathrm{a}, \mathrm{b}, *}$, Luigi Coppola ${ }^{\mathrm{a}}$, Cesare Oliviero Rossi ${ }^{\mathrm{a}}$, Giuseppe Antonio Ranieri ${ }^{\mathrm{a}}$ \\ a Department of Chemistry, University of Calabria, Ponte P. Bucci, Cubo 14/D, 87036 Arcavacata di Rende, Cosenza, Italy \\ ${ }^{\mathrm{b}}$ Department of Chemical Engineering, University of Coimbra, 3030-290 Coimbra, Portugal
}

\section{A R T I C L E I N F O}

\section{Article history:}

Received 1 April 2008

Received in revised form 30 May 2008

Accepted 9 June 2008

Available online xxx

\section{Keywords:}

Biovesicle

$k$-Carrageenan

HMCMC

Carboxymethyl cellulose

Sodium oleate

Monoolein

Networks

\begin{abstract}
A B S T R A C T
Vesicles composed of sodium oleate $(\mathrm{NaO})$ and monoolein (MO) are adequate candidates for drug nanoencapsulation and controlled release due to their stability and perceived biocompatibility. The object of the present study is to design hydrogels based on those anionic vesicles and polymers of both non-associative and associative type. The selected macromolecules were $k$-carrageenan (KC), carboxymethyl cellulose (CMC) and hydrophobically modified carboxymethyl cellulose (HMCMC). While the polymer-vesicle association was probed by rheology, the influence of the polymer on the vesicle stability was monitored by cryo-TEM and calorimetric measurements.

The effects of the polymer on the rheological properties of surfactant aggregate solutions clearly depend on the polymer type: the storage moduli of the polymer-vesicle mixtures, compared to the vesicles alone, increases around 2 orders of magnitude if the polymer is non-associative and 4 orders of magnitude if the macromolecule is of associative type.

As the vesicles are added, the non-associative polymer networks tend to be disrupted, while the networks formed by associative polymer get more robust. These observations can be explained by fundamental changes in electrostatic/hydrophobic interactions: vesicles entrapped in KC networks convert the polysaccharide in a highly charged entity and favor high electrostatic repulsions between the chains; this encourages network collapse. The opposite picture is experienced in HMCMC systems, i.e., such network is stabilized by the presence of vesicles. This is ascribed to the enhanced hydrophobic association, compensating the electrostatic repulsions between vesicles and polymer chains.
\end{abstract}

(C) 2008 Elsevier B.V. All rights reserved.

\section{Introduction}

Encapsulation of both hydrophilic and hydrophobic drugs has been an important challenge for scientists. It can be achieved by entrapping the drug in a chemically crosslinked polymer matrix [1-4] or using surfactant aggregates or amphiphilic polymers for drug compartmentalization [5,6]. Physical networks have some advantages compared to those of chemical nature, mostly due to gel-liquid reversibility. Physical networks composed by vesicles and charged polysaccharides, with or without hydrophobic interactions are reported in literature [7-12]. These charged systems are considerably sensitive to salt, so that stability and collapsing of the network can be induced by electrolyte. Several reasons are behind such phenomenon: salting-out, reduction of electrostatic

\footnotetext{
* Corresponding author at: Department of Chemical Engineering, University of Coimbra, 3030-290 Coimbra, Portugal. Tel.: +351 936431 792; fax: +351239798703

E-mail address: fantunes@eq.uc.pt (F.E. Antunes).
}

repulsion [13], and the different ability of salts to form ion binding to polyelectrolytes [14].

In this study we use anionic vesicles, with long kinetic stability and perceived biocompatibility, as nanocompartments. Although some work has been reported with biocompatible systems, most of the published studies concern noncompatible molecules, used for non-biological applications or for fundamental understandings. Monoolein (MO) is biocompatible $[15,16]$ and sodium oleate $(\mathrm{NaO})$ is known to improve the biocompatibility of some nanoparticles [17]. These 2 amphiphilic molecules form different structures in water. While the critical packing parameter (CPP) of MO exceeds one, $\mathrm{NaO}$ forms normal micelles $[18,19]$. In the dilute corner, spontaneously formed vesicles with long-term stability are found (for at least 1 year) [15]. Previous studies, concerning cubic liquidcrystalline phases of MO in water, show significant time effects due to MO hydrolysis [20]. It seems that MO is more stable when present in the mixed vesicles, since no time effects were seen within 1 year. The formation of such aggregates is not dependent on preparation procedures, and the vesicles can be also formed by adding $\mathrm{NaOH}$ to an aqueous dispersion of oleic acid and MO [15]. 
Mixtures containing $1 \mathrm{wt} \%$ of $\mathrm{NaO}$ and $1 \mathrm{wt} \%$ of $\mathrm{MO}$ are near the molar stoichiometry and small unilamellar vesicles are in large excess with their size ranging from 10 to $70 \mathrm{~nm}$, mostly below $25 \mathrm{~nm}$. The situation is similar when MO content increases up to $2 \mathrm{wt} \%$. The increase of the charge density in the system by incorporation of $\mathrm{NaO}$ enhances the vesicle polydispersity, diameters ranging from 10 to $200 \mathrm{~nm}$, mostly below $40 \mathrm{~nm}$ [15].

Here we highlight on physical networks of $\mathrm{MO}-\mathrm{NaO}$ vesicles and anionic polysaccharides. The vesicles were entrapped within the polymer network and the rheological response was reported. We selected three different polysaccharides: $k$-carrageenan $(\mathrm{KC})$, carboxymethyl cellulose (CMC) and hydrophobically modified carboxymethyl cellulose (HMCMC).

\section{Materials and methods}

\subsection{Materials}

$\mathrm{NaO}$ or sodium 9-octadecenoate, is an anionic surfactant with a $\mathrm{C}_{18}$ hydrophobic chain and a carboxyl headgroup. The molecular weight is $304 \mathrm{Da}$ and was purchased from Nu-Chek Prep, Inc. (Elysian, USA) with high purity (>99\%).

MO, Rylo Mg 90 glycerol monooleate was kindly provided by Danisco Ingredients (Braband, Denmark).

$\mathrm{KC}$ is a polysaccharide, supplied by Fluka BioChemika (Buchs, Switzerland), with $M_{\mathrm{w}}$ of around $700 \mathrm{kDa}$. The basic structure of carrageenan is a linear polysaccharide made up of a repeating disaccharide sequence of $\beta$-D-galactopyranose linked through positions 1,3 (A residues) and $\alpha$-D-galactopyranose residues linked through positions 1,4 (B residues). At room temperature, the observed gelation in carrageenan is caused by helix formation $[21,22]$ and this can only occur in repeated structures where the B residue is in a1-C-4 conformation. All the gelling types of carrageenan, which include $\kappa, \beta$ and $\iota$, contain a 3,6-anhydro bridge on the B unit which forces the sugar to flip from a 4-C-1 conformation to a 1-C-4 conformation and can then form cross-link networks and gels. Formation of a helical structure is promoted by adding an appropriate electrolyte to a sufficient ionic strength and/or by lowering the temperature [23-28].

CMC, was supplied by BDH Chemicals in the sodium salt form and with low $M_{\mathrm{w}}$. CMC is fully ionized at $\mathrm{pH} 7$ and is almost nonionic at $\mathrm{pH} 2$ [29-31].

HMCMC is a semiflexible polyanion [29], in the sodium salt form; it was received as a gift by Akzo Nobel Surface Chemistry. The CMC backbone is modified by tetradecyl groups with a degree of hydrophobic substitution of $1.2 \%$ ( 1.2 C14 chains per 100 sugar units). The charge density (percentage of carboxymethyl groups) is $80 \%$ at $\mathrm{pH} 8$ ( 80 negative charges per 100 sugar units). The weight average molecular weight is around $879 \mathrm{kDa}$. The overlap concentration is around $0.06 \mathrm{wt} \%[29,30]$.

The chemicals were used without any further purification and double distilled and deionised water was used. $\mathrm{pH}$ of the samples was around 9, thus the carboxyl groups were fully ionized.

\subsection{Methods}

Vesicle stock solutions were prepared by weighting the desired amount of surfactant and water in glass ampoules containing a small magnetic stirring bar. The polymer was added afterwards to the vesicle solution. The samples were sealed, mixed in a vortex, and homogenized at $80^{\circ} \mathrm{C}$. The mixtures were stored for 1 week before measurements. To analyze their textures, samples were observed in a polarized microscope.

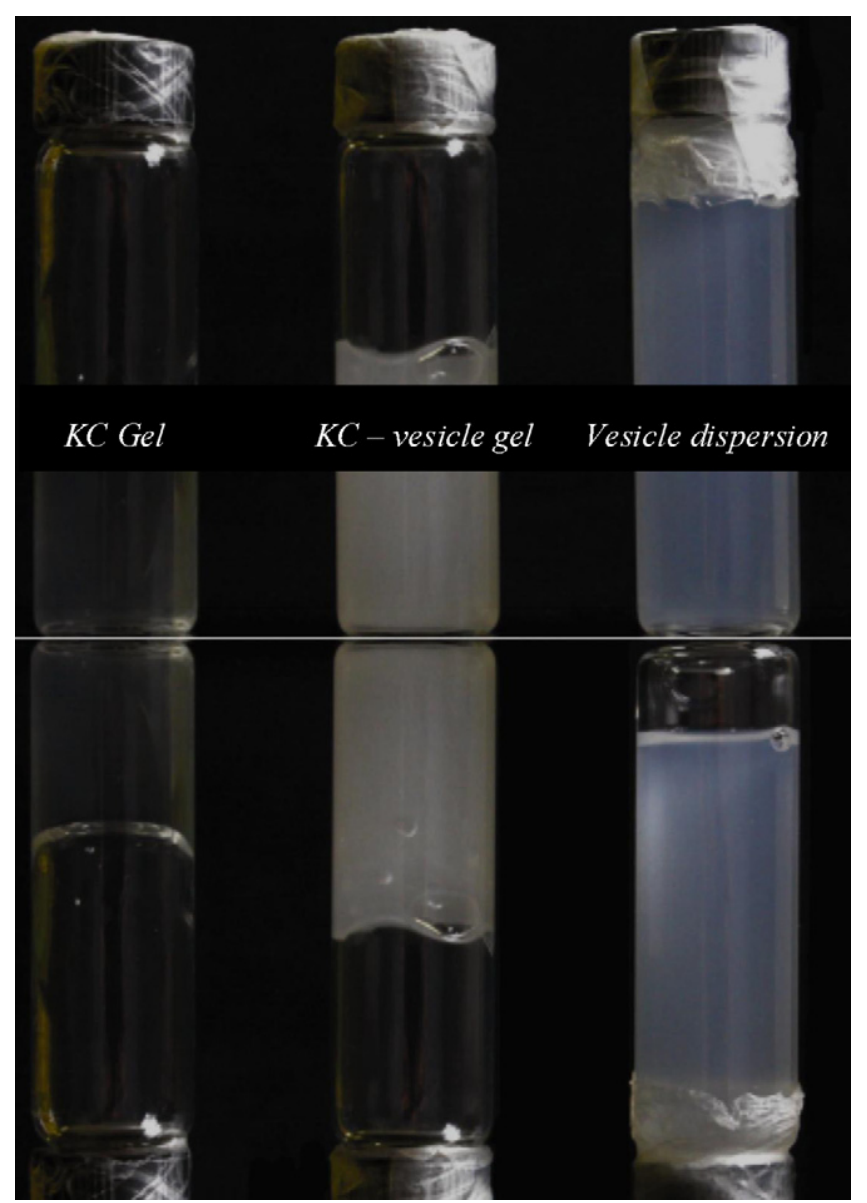

Fig. 1. Pictures of selected samples; from left to right: $0: 0: 2 \mathrm{KC}, 1: 1: 2 \mathrm{KC}$ and $1: 1: 0$.

The prepared mixtures were labeled following the nomenclature " $a: b: c P$ ", being $a$ the concentration of $\mathrm{NaO}$ (wt\%), $b$ the concentration of $\mathrm{MO}$ (wt\%), $c$ the concentration of polysaccharide (wt\%), and $P$ the polysaccharide name.

The experiments were performed far from the melting transition of the surfactants alkyl chains to prevent the network properties to be influenced by the different states [32].

While the sample containing only $\mathrm{NaO}$ is transparent, which results from the micellar phase reported in the literature $[18,19]$, the remaining samples exhibit bluish color, which is usually related to the existence of vesicles.

Polymer-free solutions are liquid-like but when polymer is added, a gel-like behavior is observed (if polymer solution is intrinsically a gel). Fig. 1 shows selected images of the samples.

Rheological measurements were conducted using a shear strain controlled rheometer RFS III (Rheometrics, USA) equipped with a couette and a microcouette, cylinder geometry (gap $1.06 \mathrm{~mm}$, inner radius 17 and $7 \mathrm{~mm}$, respectively), according to the viscosity of the samples. The temperature was controlled by a water circulator apparatus $\left( \pm 0.5^{\circ} \mathrm{C}\right)$. To prevent errors due to evaporation, the measuring geometries were surrounded by a solvent trap containing water. Two different kinds of experiments were carried out: (a) steady flow experiments were performed in a shear rate range $0.02-1700 \mathrm{~s}^{-1}$. Sometimes it was not possible to cover all shear rate range due to the instrumental limits imposed by the viscosity of the samples. The minimum and the maximum torque that the instrument can measure are 0.04 and $1000 \mathrm{~g} \mathrm{~cm}$, respectively. To be sure that the samples were in steady flow, the flow equilibrium time was measured by transient experiments (step-rate test) and 


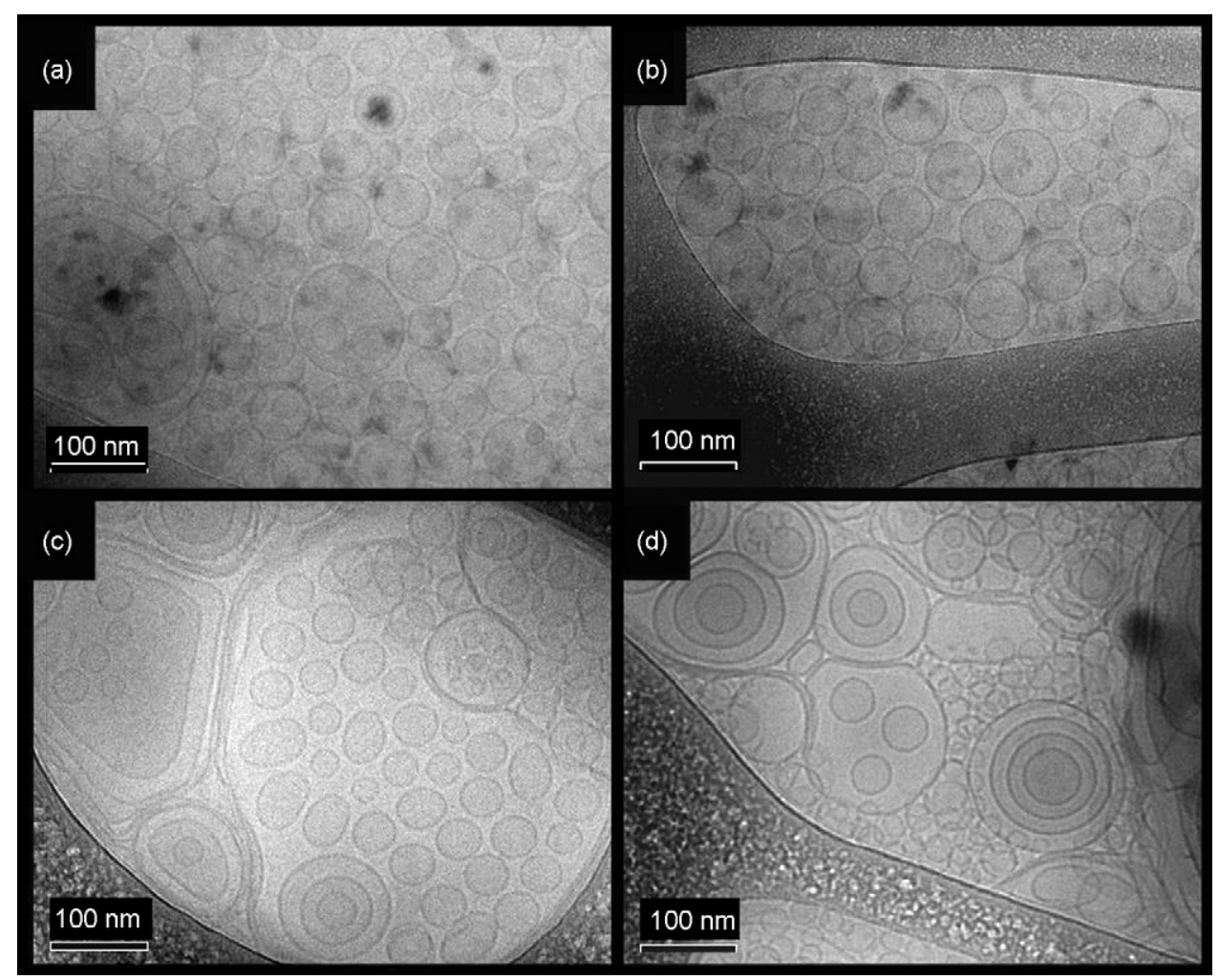

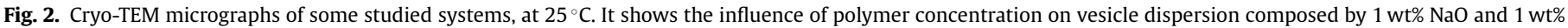
MO. Micrographs (a) and (b) refer to $0.5 \mathrm{wt} \%$ HMCMC (1:1:0.5HMCMC), while (c) and (d) refer to 1 wt\% of HMCMC (1:1:1HMCMC).

it was observed that $10 \mathrm{~s}$ was a sufficient time to ensure the steady flow in the system for the overall investigated shear rate range. (b) Dynamic shear experiments were performed in a frequency range between 0.1 and $15.9 \mathrm{~Hz}$. The small-amplitude dynamic tests provided information on the linear viscoelastic behavior of materials through the determination of the complex shear modulus [33].

$G *(\omega)=G^{\prime}(\omega)+i G^{\prime \prime}(\omega)$

where $G^{\prime}(\omega)$ is the in-phase (or storage) component and $G^{\prime \prime}(\omega)$ is the out-of-phase (or loss) component. $G^{\prime}(\omega)$ is a measure of the reversible, elastic energy, while $G^{\prime \prime}(\omega)$ represents the irreversible viscous dissipation of the mechanical energy. The dependence of these quantities on the oscillating frequency gives rise to the socalled mechanical spectrum, allowing the quantitative rheological characterization of studied materials.

Weak gel model was applied to some of the oscillatory spectra [34,35]:

$|G *(\omega)|=\sqrt{G^{\prime}(\omega)^{2}+G^{\prime \prime}(\omega)^{2}}=A \omega^{1 / z}$

where " $A$ " is interpreted as the interaction strength between the rheological units, a sort of amplitude of cooperative interactions, and " $z$ " as the coordination number, which corresponds to the number of flow units interacting with each other to give the observed flow response.

Cryogenic transmission electron microscopy (cryo-TEM) studies were performed using a Philips CM120 Bio-twin microscope. Samples were placed in a vitrification chamber in which temperature and humidity can be controlled. In particular, humidity can be held close to saturation to prevent sample evaporation. A small drop was put on a copper grid supported carbon coated holey film. Immediately after blotting with filter paper to obtain a thin liquid film over the grid, the sample is plunged into liquid ethane (at its melting temperature). The vitrified film is then transferred under liquid nitrogen to the electron microscope.

Differential scanning calorimetry (DSC) measurements were done in a Setaram DSC-131 instrument. A $20-30 \mathrm{mg}$ portion of the sample was weighed in crucibles of $1 \mathrm{~cm}^{3}$ capacity. The samples were sealed in aluminum pans. As a reference, a sealed pan with the corresponding amount of water was used. To check water evaporation, the pans were weighed before and after the DSC measurements. The DSC thermograms were recorded in the temperature range from 5 to $80^{\circ} \mathrm{C}$. The heating rate was $1^{\circ} \mathrm{C} \mathrm{min}-1$.

\section{Results and discussion}

\subsection{Polymer impact in vesicle stability}

The macromolecules used here promote longer stability of the vesicles. Those vesicles are kinetically stable in polymer free solutions and thermodynamic equilibrium conditions occur in a heterogeneous mixture of vesicles and lamellar phases [20]. Since almost all the polymer concentrations used here are above the overlap concentration, we assume that the vesicles stay entrapped in the polymer network, which delays or even prevents phase separation. Support comes from repeated visual checks over one year. Fig. 2(a-d) shows cryo-TEM micrographs of vesicles in presence of one of the polysaccharides, HMCMC. It indicates that vesicles size is not much affected by the presence of polymer: the diameter ranges from 10 to $200 \mathrm{~nm}$ both with and without polymer.

Vesicle stability was also checked by DSC and the results can be seen in Fig. 3. No vesicle phase transition was detected within the studied temperature range, indicating that vesicles are stable. A typical scan of a selected $\mathrm{KC}$-vesicle mixture is shown, and the plot shows the occurrence of an intense and sharp peak, ascribed to the polymer helix-coil transition [36,37]. 

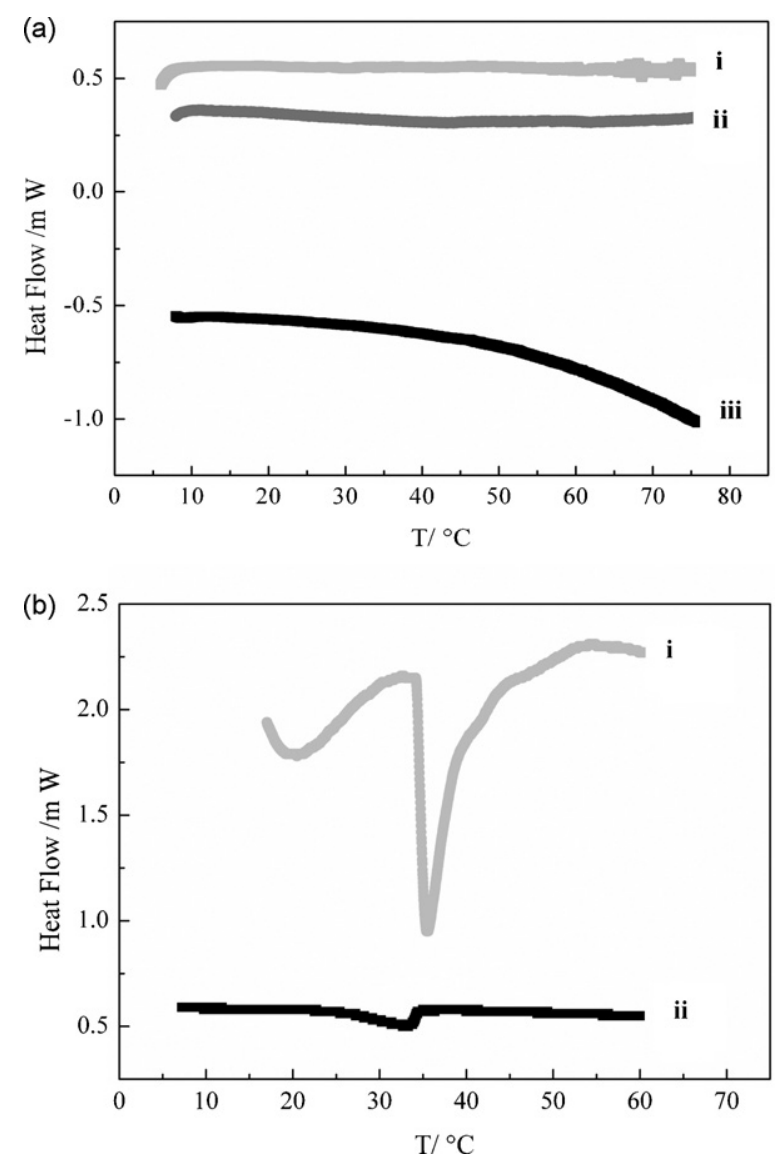

Fig. 3. (a) DSC upscans of selected HMCMC-vesicle samples: (i) 1:0.5:0, (ii) 1:1:0.5HMCMC, (iii) $1: 2: 1 \mathrm{HMCMC}$ (b) DSC upscans of selected KC-vesicle samples: (i) $0: 0: 2 \mathrm{KC}$, (ii) $1: 1: 2 \mathrm{KC}$. Heating rate: $1.0^{\circ} \mathrm{C} / \mathrm{min}$.

\subsection{KC-vesicle complexes}

Fig. 4 shows the rheological behavior of $\mathrm{KC}$, both in vesiclefree solutions (Fig. 4(a)) and in the mixed polymer-vesicle systems (Fig. 4(b)). Fig. 4(a) indicates that the rheological response changes from liquid to solid by increasing polymer concentration from 0.1 to $2 \%$, at $25^{\circ} \mathrm{C}$. This can be understood as a transition from dilute to semi-dilute regime, since the polymer overlap concentration is within 0.2 and $0.4 \mathrm{wt} \%$ [36].

While the vesicle dispersion alone has a liquid-like response, the addition of polymer, shown in Fig. 4(b), has markedly differences: the vesicle system acquires the gel-like properties of the polyelectrolyte alone. The storage modulus of the vesicle dispersion increases at least 2 orders of magnitude (depending on frequency of observation), even at $1 \mathrm{wt} \%$ of polymer. If the polymer concentration increases to $2 \mathrm{wt} \%$, the storage modulus reaches 4 orders of magnitude more than the one of vesicles alone.

By looking at the polymer network as the reference, it is weakened with the addition of the surfactant aggregates, independently if $\mathrm{KC}$ is in helix or coil conformation. Fig. 5 illustrates the influence of the vesicles on the rheological response of $\mathrm{KC}$ system, at $25^{\circ} \mathrm{C}$.

Storage moduli and relaxation times decrease with surfactant addition. While the former are related to the density of active links, the latter are associated with the strength of those active links. The relaxation spectra obtained for 0:0:1 KC and 0:0:2 KC are strongly dominated by relaxation times in the order of $1-100 \mathrm{~s}$. However, on the time scale of few milliseconds, a second region of relaxation times seems to be present. In accordance with the analysis on the relaxation processes, this region refers to the local motion of a part of the polymer chain of the order of the entanglement length The addition of vesicles affects the relaxation spectra: the importance of the long relaxation times (1-100 s region) becomes lower. Therefore, the addition of vesicles inhibits the polymer association and weakens the contacts, which might be due to an electrostatic penalty. The negative charge of the surfactant aggregate plays a counter-effect against the polymer network formation; when vesicles are added, the electrostatic repulsion increases and this results in a minor ability for polymer association, which can be illustrated by a weaker rheological response. Therefore, although the polymer itself forms a network, the presence of a macroion, like a vesicle, of the same charge of the polymer opposes to the network formation. Our electrostatic-based explanation may be connected to the effect of adding iodide to polyelectrolytes [14]. The addition of salt plays an effective screening on the electrostatic repulsion and the viscosity is tremendously increased. For instance, the storage modulus increases ca. 3 orders of magnitude by adding $1 \mathrm{M}$ of sodium chloride to the $1: 1: 1 \mathrm{KC}$ sample.

The effect of temperature has a strong impact in the dynamics of the system. The network becomes weaker as the temperature rises from 25 to $37^{\circ} \mathrm{C}$. When the temperature decreases, a coil-helix transition occurs, manifesting itself as a sol-gel transformation. This transition is thermally reversible. Calorimetric measurements showed that the KC-containing gel (semidiluted region) melts around $33^{\circ} \mathrm{C}$. An abrupt decrease in the storage modulus observed at $30-40^{\circ} \mathrm{C}$, shown in Fig. 6 for $\mathrm{KC}$-vesicle mixture, well correlates with the temperature of the helix-coil transition in $\mathrm{KC}[36,37]$,
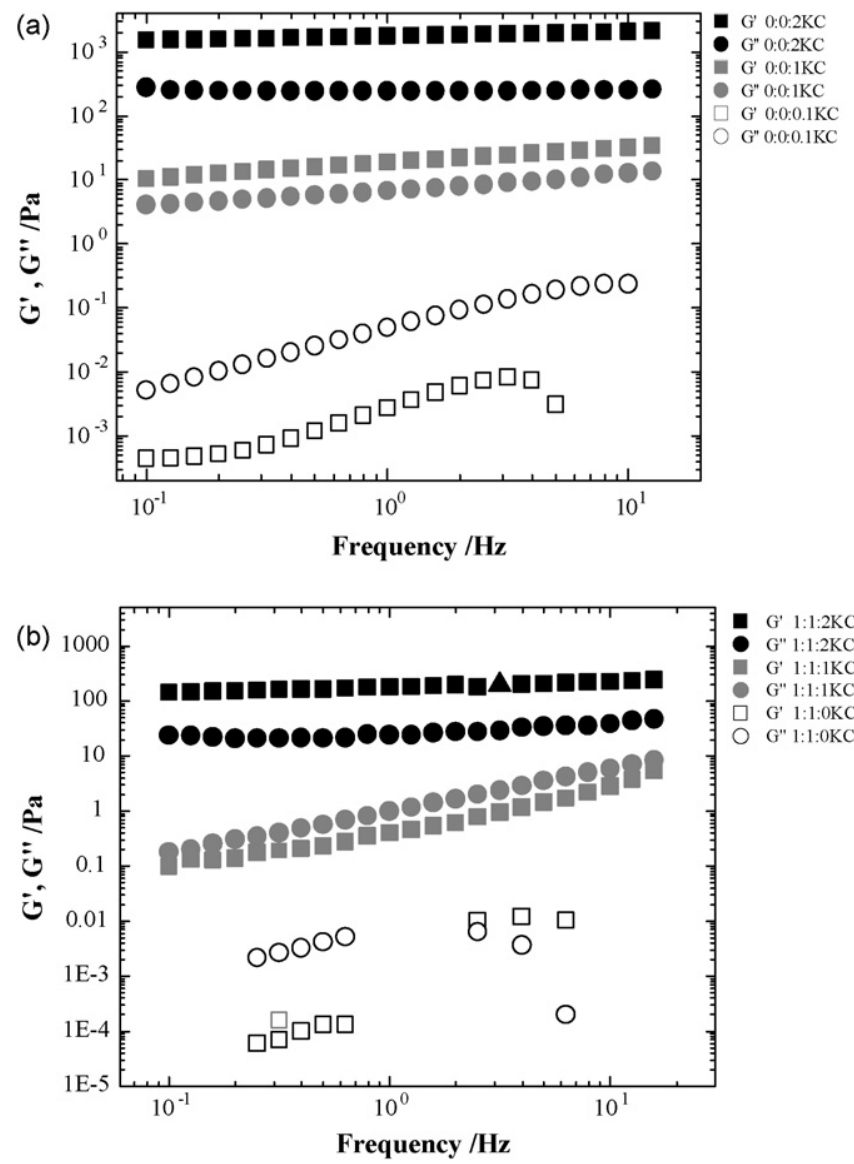

Fig. 4. (a) Frequency dependence of the storage modulus $\left(G^{\prime}\right)$ and loss modulus $\left(G^{\prime \prime}\right)$ at different KC concentrations (b) Frequency dependence of the storage modulus $\left(G^{\prime}\right)$ and loss modulus $\left(G^{\prime \prime}\right)$, for different polymer concentrations in polymer-vesicle mixtures. All mechanical spectra are performed at $25^{\circ} \mathrm{C}$. 

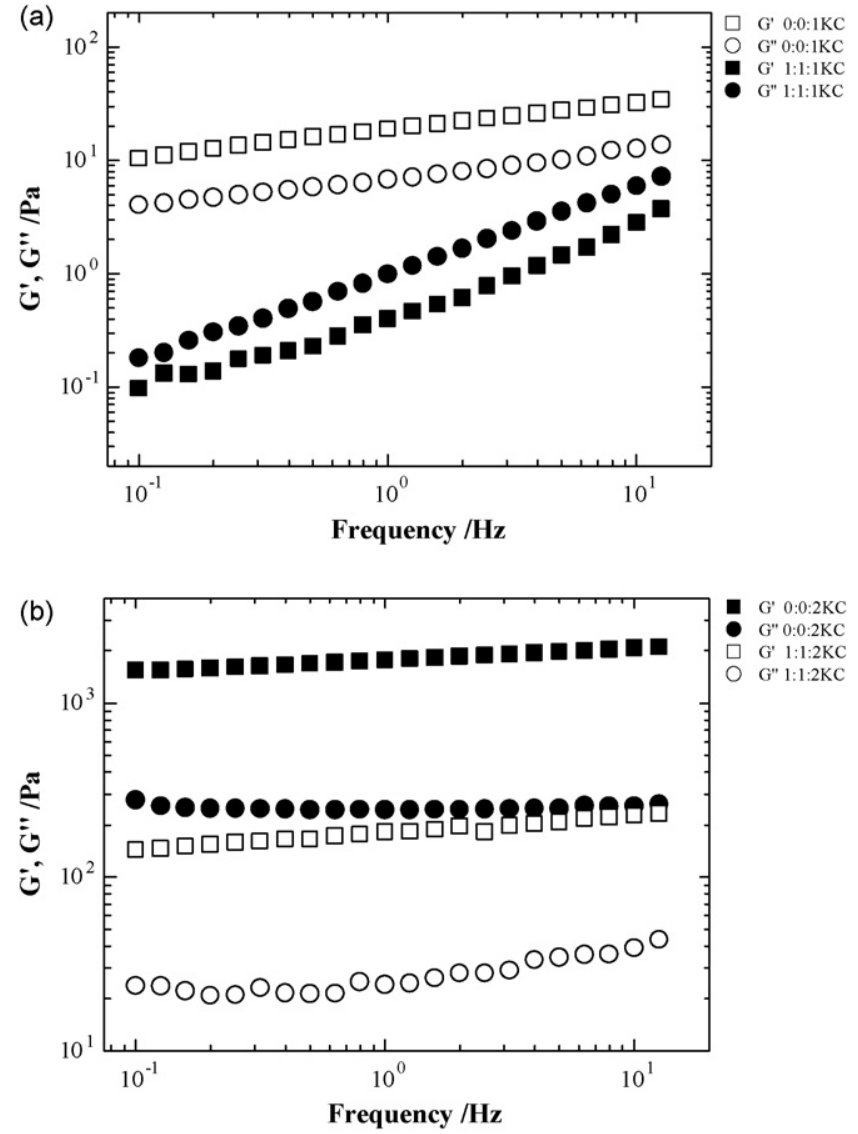

Fig. 5. (a and b) Frequency dependence of the storage modulus $\left(G^{\prime}\right)$ and loss modulus $\left(G^{\prime \prime}\right)$, for different surfactant concentration in KC-vesicle mixtures, at $25^{\circ} \mathrm{C}$.

thus suggesting that the crosslinks formed by the double helixes also take part in the formation of gel structure in this surfactantcontaining mixed system.

Fig. 7 gives additional information about the rheological properties of the studied systems at different temperatures. The effects of the vesicle composition on the rheological properties of the polysaccharide underline the mechanisms discussed above.

This figure shows the composition and temperature dependence of weak gel model parameters, $z$ and $A$, as obtained by fitting the viscoelastic data in Eq. (2) for the KC mixtures.

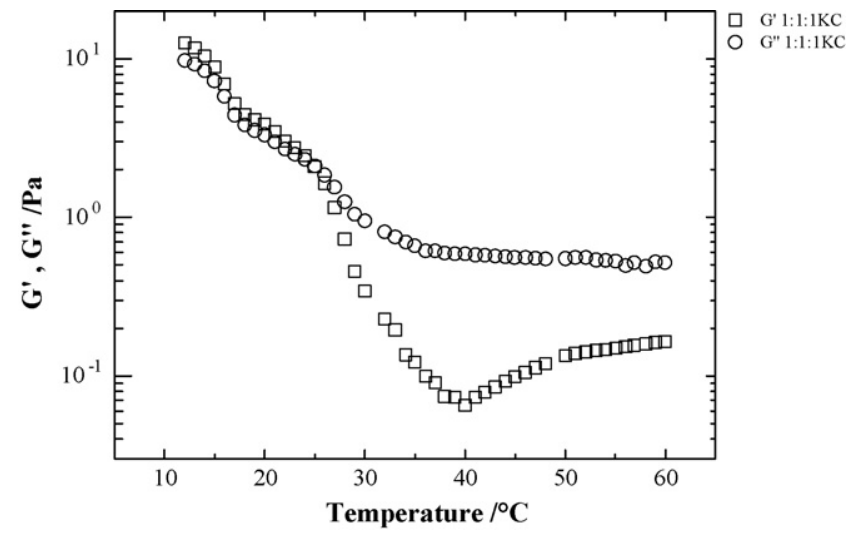

Fig. 6. Linear viscoelastic thermogram for $1: 1: 1 \mathrm{KC}$ mixtures. Temperature scan of storage modulus $G$ (circle symbols), loss modulus $G^{\prime \prime}$ (square symbols) obtained by a time-cure test frequency $=1 \mathrm{~Hz}$. Heating rate of the experiment was of $0.5^{\circ} \mathrm{C} / \mathrm{min}$ and the equilibration time of $2 \mathrm{~s}$.

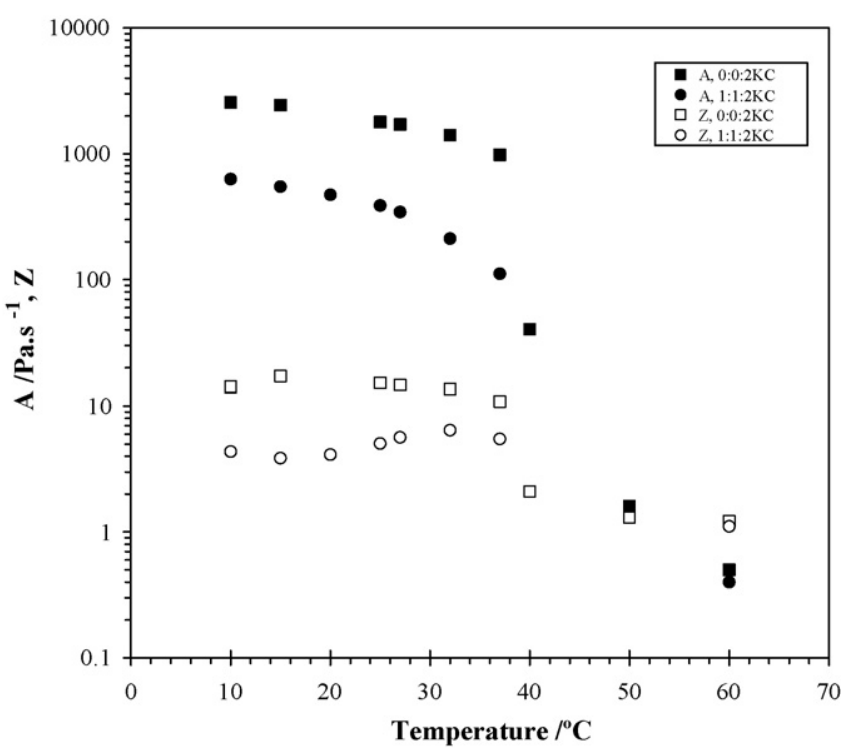

Fig. 7. Weak gel model parameters for KC systems, at different temperatures.

The surfactant-free solution shows a high flow coordination number, $z$, and high interaction strength values, $A$, in the temperature range $10-60^{\circ} \mathrm{C}$. It is worth noticing that the $A$ values decrease with the temperature, while the $\mathrm{z}$ number appears rather constant, which means a weakening of the gel network but not a decreasing of the flow units. The $A$ and $z$ parameters of vesicles-KC mixtures are always lower than the ones of binary $\mathrm{KC}$-water mixtures.

\subsection{HMCMC and $C M C$ - vesicle complexes}

Fig. 8 shows the influence of $\mathrm{HMCMC}$ on the rheology of $\mathrm{NaO} / \mathrm{MO}$ vesicles.

The rheological behavior changes from liquid to solid by adding HMCMC to vesicle dispersion and thus the vesicles are entrapped in the polymer entanglements. The vesicle storage modulus increases at least 4 orders of magnitude (depending on frequency) if $1 \mathrm{wt} \%$ of polymer is added.

Fig. 9 shows the influence of surfactant concentration on the HMCMC network properties. The behavior can be reported as an increase in elastic response by addition of the vesicles and clearly differs from the behavior found above for $\mathrm{KC}$.

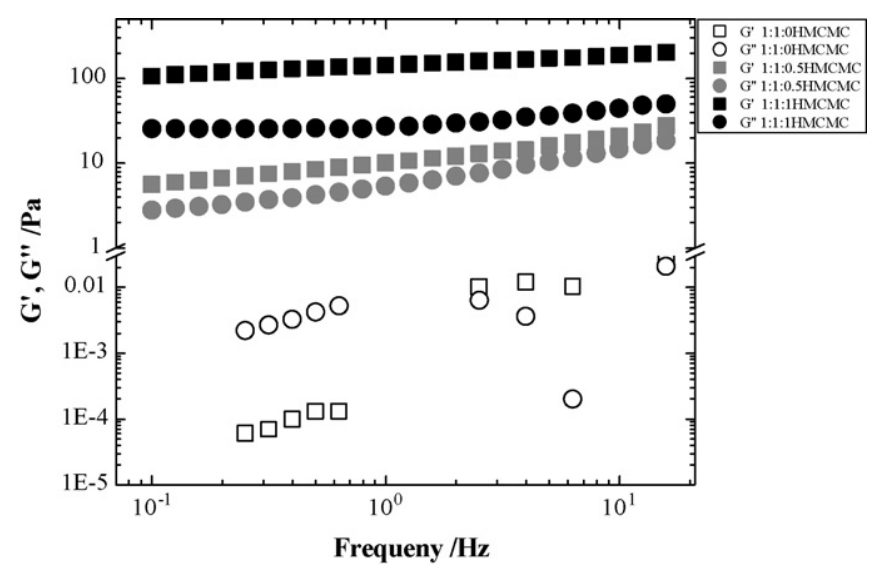

Fig. 8. Frequency dependence of the storage modulus $\left(G^{\prime}\right)$ and loss modulus $\left(G^{\prime \prime}\right)$, at different polymer concentrations in HMCMC-vesicle mixtures, at $25^{\circ} \mathrm{C}$. 


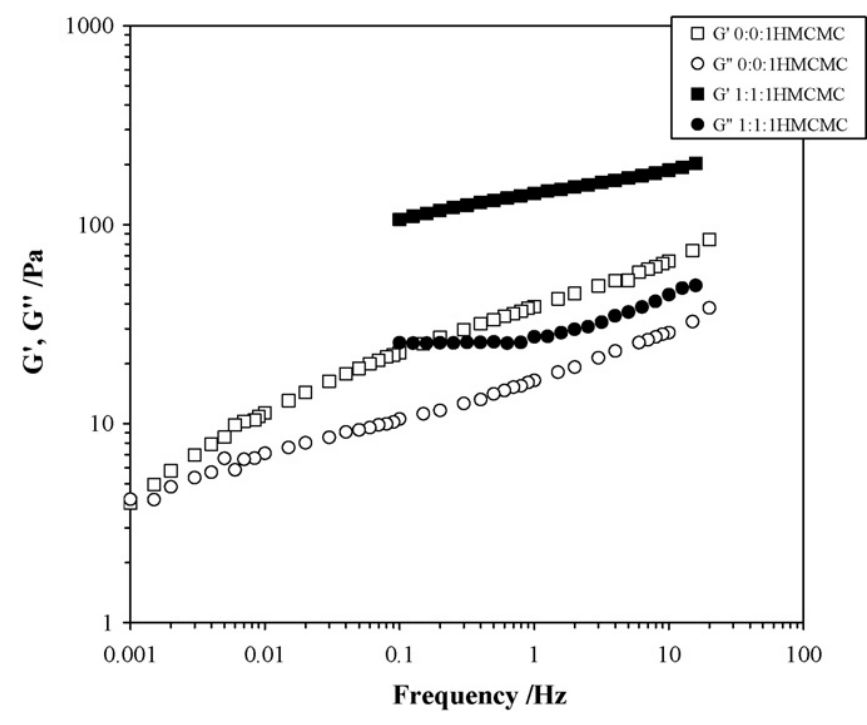

Fig. 9. Frequency dependence of the storage modulus $\left(G^{\prime}\right)$ and loss modulus $\left(G^{\prime \prime}\right)$, at different surfactant concentration in HMCMC-vesicle mixtures, at $25^{\circ} \mathrm{C}$.

As a control system, the rheology of CMC-vesicle system was also monitored and shown in Fig. 10(a and b). This system has particular importance to understand the role of the hydrophobic modification. While the increase in the storage modulus of vesicle
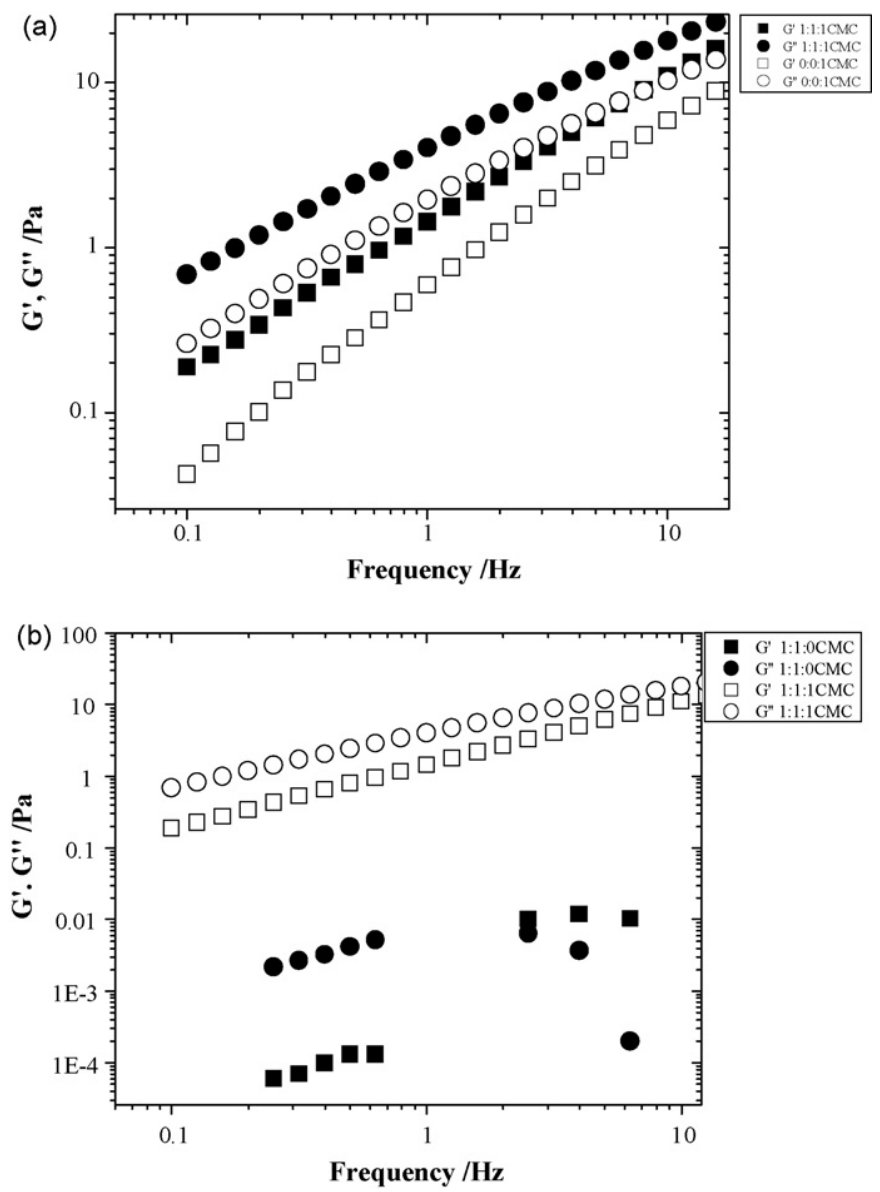

Fig. 10. Frequency dependence of the storage modulus $\left(G^{\prime}\right)$ and loss modulus $\left(G^{\prime \prime}\right)$, indicating the influence of the polymer on the vesicle rheological response (a) and the influence of the surfactants on the polymer viscoelasticity (b), at $25^{\circ} \mathrm{C}$.

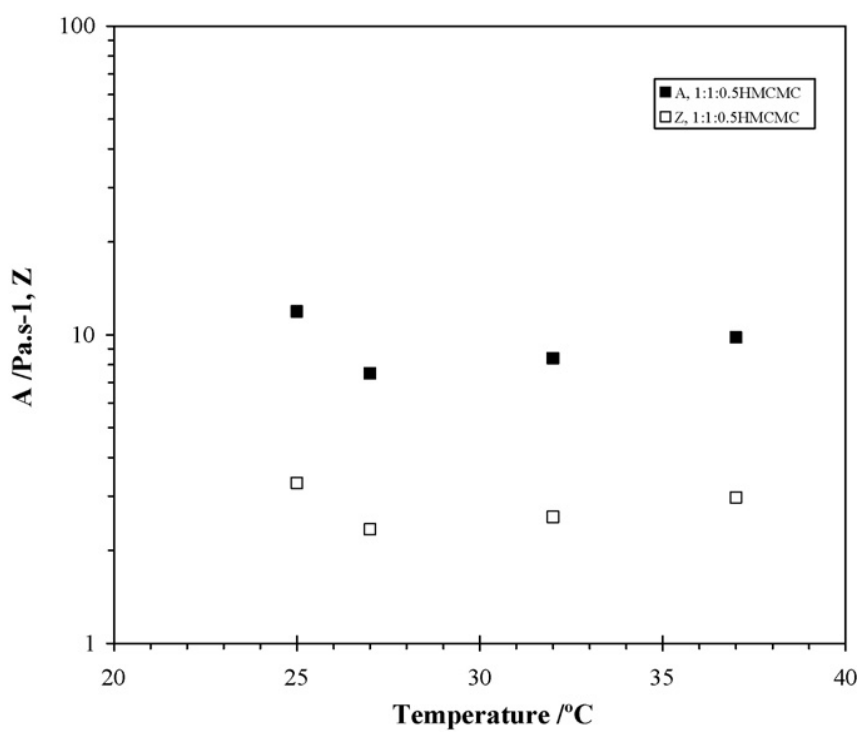

Fig. 11. Weak gel model parameters for HMCMC systems, at different temperatures.

system due to the presence of CMC is ca. 2 orders of magnitude, this value reaches 4 if the macromolecule is hydrophobically modified. The differences can be addressed to the polymer composition and to the HMCMC hydrophobic capability for association, and this plays a strong driving force for polymer-vesicle interaction.

Fig. 10(b) indicates that CMC solution become more elastic when vesicles are added. Such behavior is different from the one observed in KC mixtures. It is plausible to think that the electrostatic penalty, due to the addition of surfactant aggregates of the same charge, is stronger when the polymer solution is a gel. When in dilute regimes (well below the overlap concentration), the inter-chain distance is large and the addition of a similarly charged compound increases the viscosity due to an effect similar to the Einstein equation for dilute dispersion of spherical particles [38]. This picture is, however, the opposite of the observed above, when the vesicle dispersion is added to a semi-dilute polymer (KC) solution.

Weak gel models were applied to the HMCMC results, at different temperatures, and are resumed in Fig. 11. While the ternary surfactant system shows $z$ values close to the unit and $A$ values with an order of magnitude of $10^{-3}$, the surfactant-polymer systems show $A$ and $z$ values of an order of magnitude of $10^{2}$ and 10 , respectively.

The results described above show that the biofriendly vesicles NaO-MO-W can be entrapped in polymer networks, which have manifestly high potential for controlled drug and gene release. The viscosity, and thus the release rate, may be tuned by the presence of hydrophobic groups in the polymer chain.

\section{Conclusions}

Vesicles composed by $\mathrm{NaO}$ and $\mathrm{MO}$ were mixed with both associative and non-associative anionic polysaccharides, resulting in gels with interesting rheological properties. The dynamic properties of the mixed systems can be both more elastic and more liquid than the polymer solutions alone, depending if the polymer is of associative or non-associative type, respectively, i.e., vesicles may increase the polymer gel consistence or favor its disruption if vesicle-polymer hydrophobic interactions are present. Within the used concentrations, the polymer itself forms a network and the presence of a macroion of the same charge, as a vesicle, opposes to the network formation. The electrostatic repulsion inhibits the interaction between the chains and the entanglements become 
weaker. The incorporation of hydrophobic groups in the polymer stabilizes the networks due to effective hydrophobic crosslinks between the polymer and the surfactant aggregate.

\section{Acknowledgments}

FEA acknowledges Fundação para Ciência e Tecnologia (FCT) for a research grant (ref. SFRH/BPD/26801/2006). Adelino Abreu and Luigi Gentile are acknowledged for technical assistance. We thank Prof. Björn Lindman for fruitful discussions, Leif Karlson at Akzo Nobel Surface Chemistry, Stenungsund, Sweden, for supplying HMCMC, and Danisco Ingredients, Denmark, for supplying MO. We are grateful to the Biomicroscopy Unit at the Chemical Center, Lund University, Sweden.

\section{References}

[1] L.J. Bee, J. Yoon, D.S. Lee, T.G. Park, J. Biomater. Sci. Polym. Ed. 15 (2004) 1571-1583.

[2] M. Ishihara, M. Kikuchi, J. Artif. Organs 9 (2006) 8-16.

[3] J.B. Leach, C.E. Schmidt, Biomaterials 26 (2005) 125-135.

[4] N.A. Peppas, J.Z. Hilt, A. Khademhosseini, R. Langer, Adv. Matter 18 (2006) $1345-1360$.

[5] M. Guedeau-Boudeville, L. Jullien, J. Meglio, Biophys. J. 92 (2005) 9590-9592.

[6] B. Yu, T. Okano, K. Kataoka, G. Kwon, J Control. Release 53 (1998) 131-136.

[7] F.E. Antunes, E.F. Marques, R. Gomes, K. Thuresson, B. Lindman, M.G. Miguel, Langmuir 20 (2004) 4647-4656.

[8] F. Bordi, C. Cametti, M. Diociaiuti, D. Gaudino, T. Gili, S. Sennato, Langmuir 20 (2004) 5214-5222.

[9] J. Kevelam, J.F.L. vanBreemen, W. Blokzijl, J. Engberts, Langmuir 12 (1996) 4709-4717.

[10] J.H. Lee, J.P. Gustin, T.H. Chen, G.F. Payne, S.R. Raghavan, Langmuir 21 (2005) 26-33.

[11] K. Loyen, I. Iliopoulos, R. Audebert, U. Olsson, Langmuir 11 (1995) 1053-1056.

[12] O. Regev, E.F. Marques, A. Khan, Langmuir 15 (1999) 642-645.
[13] F.E. Antunes, B. Lindman, M.G. Miguel, Langmuir 21 (2005) 10188-10196

[14] M. Rinaudo, Macromol. Biosci. 6 (2006) 590-610.

[15] J. Borné, T. Nylander, A. Khan, J. Coll. Interface Sci. 257 (2003) 310-320.

[16] A. Ganem-Quintanar, D. QUintanar-Guerrero, P. Buri, Drug Dev. Ind.Pharm. 26 (2000) 809-820.

[17] J. Sun, S. Zhou, P. Hou, Y. Yang, J. Weng, X. Li, M. Li, J. Biomed. Mater. Res. Part A 80 (2007) 333-341.

[18] F.E. Antunes, L. Coppola, D. Gaudio, I. Nicotera, C. Oliviero, Colloids Surf. A 297 (2007) 95-104.

[19] R.D. Vold, J. Chem. 43 (1939) 1213

[20] J. Borné, T. Nylander, A. Khan, Langmuir 17 (2001) 7742.

[21] A. Hugerth, S. Nilsson, L.O. Sundelöf, Int. J. Biol. Macromol. 26 (1999) 69-76.

[22] M.T. Nickerson, A.T. Paulson, Carbohydr. Polym. 61 (2005) 231-237.

[23] L. Piculell, Food Polysaccharides and Their Applications, CRC Press, New York, 1995.

[24] G.H. Therkelsen, Industrial Gums: Polysaccharides and Their Derivatives, Academic Press, Inc., San Diego, 1993.

[25] J.M. Guenet, Thermoreversible Gelation of Polymers and Biopolymers, Academic Press, London, 1992.

[26] E.R. Morris, I.T. Norton, Stud. Phys. Theor. Chem. 26 (1983) 549.

[27] V.J. Morris, Functional Properties of Food Macromolecules, Elsevier, London, 1986.

[28] K. Te Nijenhuis, Adv. Polym. Sci. 130 (1997) 1.

[29] C.W. Hoogendam, A. Keizer, M.A. Cohen Stuart, B.H. Bijsterbosh, Macromolecules 31 (1998) 6297-6309.

[30] C. Alvarez-Lorenzo, R. Duro, J.L. Goméz-Amoza, R. Martínez-Pacheco, C.C. Souto, Colloid Polym. Sci. 279 (2001) 1045-1057.

[31] M.A. Cohen Stuart, R.G. Fokkink, P.M. van der Horst, J.W. Th Lichtenbelt, Colloid Polym. Sci. 276 (1998) 335-341.

[32] F.E. Antunes, R.O. Brito, E.F. Marques, B. Lindman, M. Miguel, J. Phys. Chem. B 111 (2007) 116-123.

[33] J. Ferry, Viscoelastic Properties of Polymers, 3rd ed., John Wiley \& Sons, New York, 1980.

[34] D. Gabriele, B. De Cindio, P. D’Antona, Rheol. Acta 40 (2001) 120.

[35] L. Coppola, R. Gianderri, I. Nicotera, C. Oliviero, G.A. Ranieri, Phys. Chem. Chem. Phys. 6 (2004) 2364-2372.

[36] M.T. Nickerson, A.T. Paulson, Carbohydr. Polym. 58 (2004) 15-24.

[37] M.P. Gonçalves, C. Gomes, Biopolymers 41 (1998) 657-671.

[38] K. Holmberg, B. Jönsson, B. Kronbreg, B. Lindman, Surfactants and Polymers in Aqueous Solution, 2nd ed., John Wiley \& Sons, 2003. 